\title{
Frontend light source for short-pulse pumped OPCPA system
}

\author{
I. Ahmad $\cdot$ S.A. Trushin $\cdot$ Z. Major $\cdot$ C. Wandt • \\ S. Klingebiel · T.-J. Wang • V. Pervak • A. Popp • \\ M. Siebold • F. Krausz $\cdot$ S. Karsch
}

Received: 20 April 2009 / Published online: 5 June 2009

(C) The Author(s) 2009. This article is published with open access at Springerlink.com

\begin{abstract}
We present the development of a light source for generating optically synchronized seed pulses both for the parametric amplifier chain and the pump-laser chain of the Petawatt Field Synthesizer (PFS), which is currently under construction at MPQ. The PFS system aims at delivering waveform-controlled few-cycle laser pulses with PW-scale peak power using optical parametric chirped pulse amplification (OPCPA). Methods of generating the broadband nearinfrared (NIR) seed pulses for the OPCPA chain by spectral broadening using few-cycle pulses are presented. We also demonstrate the generation of a supercontinuum spanning up to three octaves $(270-1500 \mathrm{~nm})$ using cascaded hollowcore fibers which supports sub-cycle pulse duration.
\end{abstract}

PACS 52.38. $\mathrm{Hb} \cdot$ 42.65.Re $\cdot$ 42.72. Ai $\cdot 42.65 . \mathrm{Yj}$

\section{Introduction}

High-power, few-cycle light pulses are of great interest for studying laser-matter interactions at extreme conditions. A number of applications such as the generation of monoenergetic electron beams or the generation of intense single

I. Ahmad $(\bowtie) \cdot$ S.A. Trushin $\cdot$ Z. Major $\cdot$ C. Wandt .

S. Klingebiel - T.-J. Wang · V. Pervak · A. Popp · M. Siebold .

F. Krausz $\cdot$ S. Karsch

Max-Planck-Institut für Quantenoptik (MPQ),

Hans-Kopfermann-Str. 1, 85748 Garching, Germany

e-mail: izhar.ahmad@mpq.mpg.de

S.A. Trushin

e-mail: sergei.trushin@mpq.mpg.de

Z. Major · V. Pervak · F. Krausz $\cdot$ S. Karsch Ludwig-Maximilians-Universität München, Am Coulombwall 1, 85748 Garching, Germany attosecond pulses from solid density plasmas have already emerged from this field [1-5] and calls for light sources delivering ever shorter and more powerful pulses. Optical parametric chirped pulse amplification (OPCPA) [6] offers a promising route towards such high-power light sources owing to the very broad gain-bandwidth that can be achieved in the nonlinear frequency-mixing process [7]. Recently, a number of high-power OPCPA systems operating with pump pulses of $100 \mathrm{ps}-10 \mathrm{~ns}$ duration have been reported. They can deliver pulse energies as high as $35 \mathrm{~J}$ in $84 \mathrm{fs}$ [8] or $24 \mathrm{~J}$ in $43 \mathrm{fs}$ [9] pulses amplified in narrowband nonlinear DKDP crystals and $90 \mathrm{~mJ}$ in the few-cycle regime (10 fs) using BBO as the nonlinear medium [10]. However, the generation of Joule-scale pulse energies in the few-cycle regime has yet to be demonstrated and constitutes the main goal of the development of the Petawatt Field Synthesizer (PFS) infrastructure at the Max-Planck Institute for Quantum Optics (Garching, Germany).

The PFS project aims at developing a light source delivering petawatt-scale carrier-envelope phase (CEP) controlled pulses with pulse energies $>3 \mathrm{~J}$ in the few-cycle regime $(\sim 5 \mathrm{fs}, 700-1400 \mathrm{~nm})$ at a repetition rate of $10 \mathrm{~Hz}$ $[11,12]$. The design of this system is based on a modified OPCPA scheme where short pulses on the order of 1-2 ps are used for pumping an OPCPA chain. In this approach, the large bandwidth is achieved by using thin DKDP crystals, while the high-gain and pulse energies are ensured by intense pumping and large crystal size, respectively. This technique possesses immense potential for generating highcontrast pulses due to the short, ps-scale time window for the parametric fluorescence. Moreover, very simple, compact, and high throughput stretcher-compressor systems, such as bulk glass and chirped mirrors, can be used owing to the small stretching factor. However, the design of the PFS requires a sophisticated pump source delivering 1-2 ps pulses 
Fig. 1 Schematic layout of the frontend. BS: beam splitter; CMC: chirped mirror compressor; FA: fiber amplifier; HCF: hollow-core fiber; IF: interference filter; M.Ob: microscope objective; PCF: photonic crystal fiber; BBO: beta-barium borate crystal; SFG: sum frequency generation signal; HDM: high dispersive (negatively chirped) mirrors; Ar-cell: argon cell; Nano-Track: fiber output power stabilization system; Beam Lock-4D: laser beam pointing stabilization system; M: mirror; L: Lens; TS: translation stage (XYZ); FM: focusing mirror; PS: pulse selector

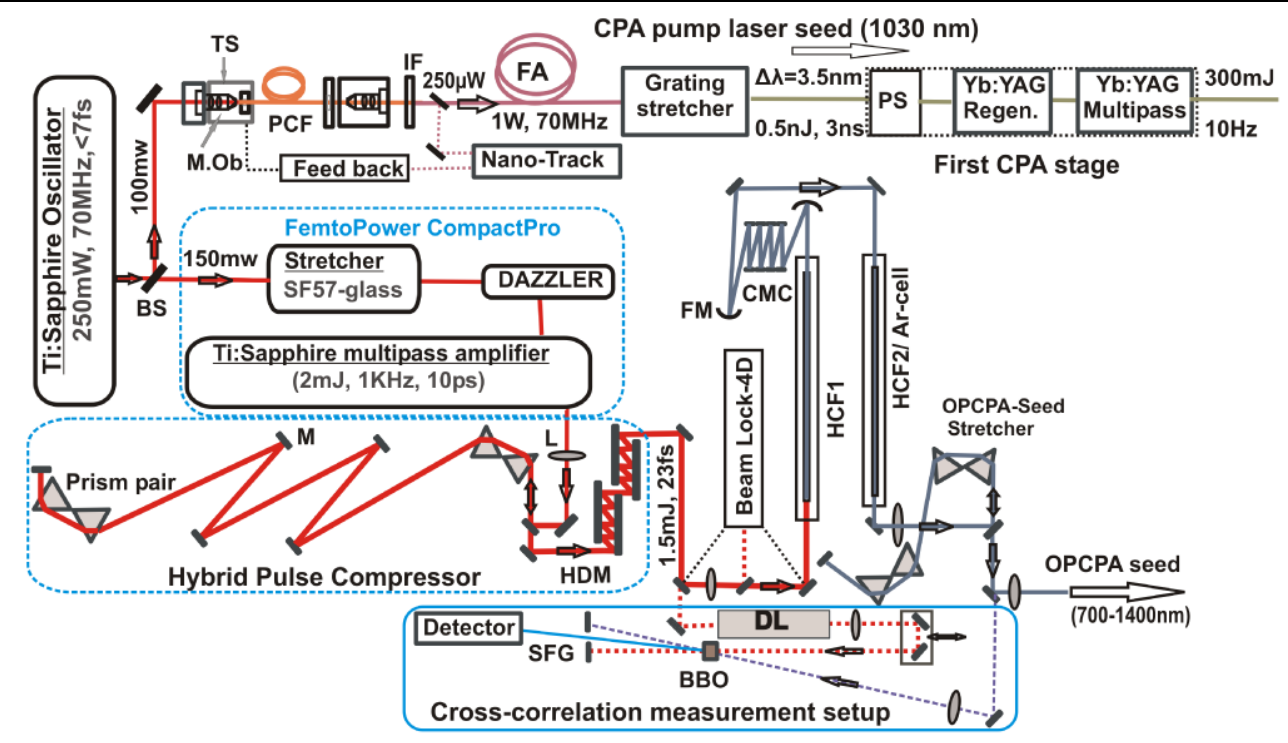

with 15-20 J (frequency doubled at $515 \mathrm{~nm}$ ) pulse energy, i.e., $50 \mathrm{~J}$ in the fundamental beam (at $1030 \mathrm{~nm}$ ), at $10 \mathrm{~Hz}$ repetition rate. Such a system is not commercially available and therefore a challenge for development on its own right. The PFS pump source with these specifications is based on the chirped-pulse amplification (CPA) principle [13] using Yb:YAG as the amplification medium pumped by highpower laser diodes [14]. In addition, the short-pulse pumped OPCPA approach demands a very accurate and stable synchronization since seed and pump pulses are only a few-ps long. This makes it necessary to derive both pulses from a common source. In the case of PFS, the pump laser requires seeding at $1030 \mathrm{~nm}$ due to the peak of the emission cross section of Yb:YAG, while the OPCPA chain needs broadband seeding in a spectral range of $700-1400 \mathrm{~nm}$ in order to achieve the largest gain bandwidth in DKDP. Furthermore, both amplifier chains need to be seeded with as energetic pulses as possible in order to limit the amplified spontaneous emission of the CPA pump-laser chain and the parametric superfluorescence of the main OPCPA chain [10, 15]. An oscillator source satisfying these conditions, however, is not commercially available to serve as a common source for direct seeding [16].

In this paper we present a detailed description on the development of a frontend light source of the PFS which delivers optically synchronized seed pulses both for the pump laser chain and the OPCPA chain. The paper is organized as follows: Firstly, we discuss the layout of the frontend, the procedures for the pump laser and OPCPA seed generation, and a technique for stretching of the latter to the ps scale. This is followed by the results of numerical simulations of the OPCPA process using the experimentally obtained seed pulse as an input. Finally, the temporal synchronization measurement between the two seed pulses is described.

\section{Frontend layout}

The PFS frontend layout is shown in Fig. 1. A Ti:Sapphire oscillator (Rainbow: Femtolasers $\mathrm{GmbH}, 70 \mathrm{MHz}, 250 \mathrm{~mW}$, pulse duration $<7 \mathrm{fs}$, spectral range: $620-1000 \mathrm{~nm}$, CEPstabilized) is used as the master oscillator (MO). The output of the MO is divided into two parts using a broadband beam splitter. One part of the output is coupled into a photonic crystal fiber (PCF) for spectral shifting to the pump laser wavelength (see Sect. 3 for details). The remainder of the MO output is further amplified in a commercial amplifier (Femtopower Compact-Pro, Femtolasers $\mathrm{GmbH}$ ) and then compressed in a hybrid pulse compressor (HPC), which will be described in Sect. 4.1. The output of the HPC is used for generation of a broadband supercontinuum by a two-stage (or cascaded) spectral broadening technique (described in Sects. 4.2-4.4). The near-infrared (NIR) spectral part of this supercontinuum is then stretched to few ps for seeding the main OPCPA chain. The stretched pulses are characterized by a cross correlation, as detailed in Sect. 4.5. The scheme of these measurements is also depicted in Fig. 1.

\section{Pump-laser seed generation}

In order to generate the pump laser seed at $1030 \mathrm{~nm}$, we have utilized soliton self-frequency shift (SSFS) in a PCF $[10,17]$. A part of the MO output $(\sim 100 \mathrm{~mW}, 1.4 \mathrm{~nJ})$ is coupled into a PCF (type NL-PM-750, Crystal Fibre Ltd., length $25 \mathrm{~cm}$, core diameter $\sim 1.6 \mu \mathrm{m}$ ) using a microscope objective as shown in Fig. 1. In order to keep the PCF output power at $1030 \mathrm{~nm}$ constant, we use an active fiber alignment system [APT Nano-Track BNT001, NanoMax301, Thorlabs]. An interference filter selects a $\Delta \lambda=10 \mathrm{~nm}$ 


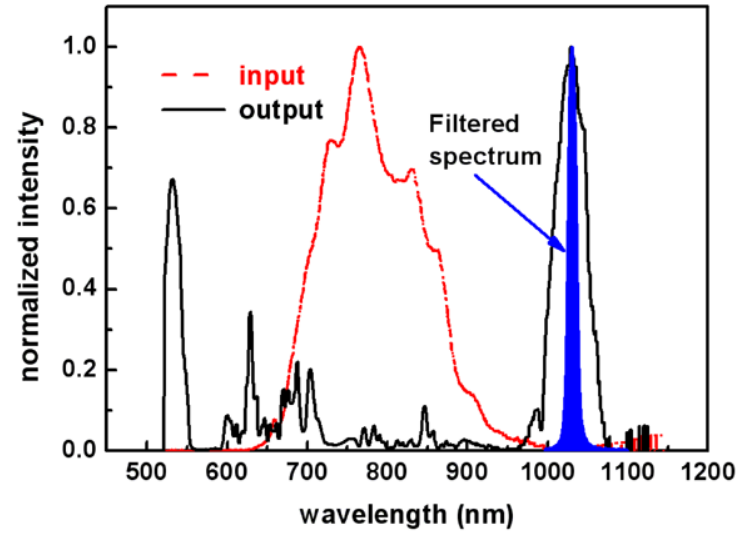

Fig. 2 Input and output spectra of the PCF. The spectrum after the interference filter is also shown in the filled area

band centered at $1030 \mathrm{~nm}$ from the frequency shifted spectrum after the PCF yielding about 3.4-pJ pulse energy after the filter (Fig. 2). This output is then coupled into an Yb-doped fiber amplifier (designed by the Institute for Applied Physics, Jena, Germany). After the fiber amplifier, 14$\mathrm{nJ}$ pulses $(70 \mathrm{MHz}, 4.4 \mathrm{ps})$ are then fed into an all-reflective grating stretcher in double-pass configuration, i.e., eight reflections off the grating [18]. At the stretcher output we get pulses with $0.5 \mathrm{~nJ}$, in $3 \mathrm{~ns}$, and a bandwidth of $\sim 4 \mathrm{~nm}$. After the stretcher these seed pulses are then amplified to $300 \mathrm{~mJ}$ at $10 \mathrm{~Hz}$ within a bandwidth of $\sim 3.5 \mathrm{~nm}$ in the first CPA stage of the pump laser consisting of an Yb:glass regenerative amplifier (RA) followed by a second Yb:YAG multipass amplifier, both pumped with laser diodes. The details of these amplifiers are described in Refs. [14, 19].

\section{OPCPA seed generation}

The generation of a broadband spectrum appropriate for seeding the main OPCPA chain of PFS (700-1400 nm) is based on the production of a supercontinuum which extends up to the NIR. A number of multiple-octave supercontinua have already been generated by the nonlinear interaction of few-cycle pulses in gaseous media [20-22]. Different cascaded spectral broadening techniques (e.g., doublefilamentation, hollow-core fiber (HCF) followed by a gascell), while having an intermediate pulse compressor have been used in these experiments. On the other hand, a doubleHCF approach was reported by Schenkel et al. [23], where generation of a broadband supercontinuum $(500 \mathrm{THz})$ and compression of a part of it to produce 3.8-fs pulses was presented. However, in all the previous experiments (according to the best of our knowledge) characterization of such supercontinua in the NIR remained undiscussed. We have carried out such investigations on two different cascaded spectral broadening schemes, namely an HCF-filament and a doubleHCF setup, and present our findings in this section. From our results it is clear that both these methods hold promise for the generation of a supercontinuum extending up to the NIR, which could then be applied for seeding an OPCPA in this spectral range. Since the broadening during such nonlinear processes demands an intensity (and hence energy) that cannot be delivered by the few-nJ scale output of the MO, a CPA scheme needs to be used. We amplified the part of the MO output which was dedicated for OPCPA seed using a CPA-based Femtopower amplifier to a pulse energy of $2 \mathrm{~mJ}$ with spectral full-width at half maximum (FWHM) of $60 \mathrm{~nm}$ at a repetition rate of $1 \mathrm{kHz}$ (Fig. 1). In the following we discuss the compression of the Femtopower output using an HPC in order to get high-quality short pulses which would then be used as the driver pulses for the generation of an ultrabroadband supercontinuum.

\subsection{Hybrid pulse compression of the Femtopower amplifier output}

The Femtopower system contains an SF57 glass-stretcher, an acousto-optical dispersive filter (DAZZLER, FASTLITE) and a 10-pass amplifier. The output of the amplifier $(2 \mathrm{~mJ}$, $\sim 10 \mathrm{ps})$ is compressed with a paired double-prism compressor as shown in Fig. 1. In every prism compressor, the pulses are first overcompressed by the negative dispersion introduced by the geometric path difference and are then finally compressed by the remaining positive dispersion in the prism material. At an energy level of our system this results in self-phase modulation (SPM) in the last prism, which in turn causes spectral narrowing (Fig. 3a) owing to the negative chirp of the laser pulse [24], and deterioration of the spatial and temporal profile of the compressed pulses. An HPC technique to overcome this problem was proposed by Cavalieri et al. [25], where the pulses after the amplifier were overcompressed by increasing the prism separation. Hence the pulses still maintain their negative chirp after the last prism and are finally compressed by the positive-dispersive mirrors (PDM). However, the use of PDM's in this HPC scheme has the drawback that the pulses pass through the last prism with negative chirp. For high energy (and hence intensity) pulses, ever more negative chirp is required to avoid SPM. Therefore the required prism separation quickly becomes inconvenient and may even lead to spectral clipping in the second pair of prisms. Moreover, changing the prism separation also results in changing the higher-order dispersion components, which in turn requires additional dispersion management.

The advent of negatively chirped high dispersive mirrors (HDM) [26], with sufficiently high dispersion to overcompensate the positive dispersion of the last prism pair, have made it possible to compress high-energy pulses in a straightforward way in HPC, i.e., maintaining the positive chirp of the pulses after the prism compressor and finally 


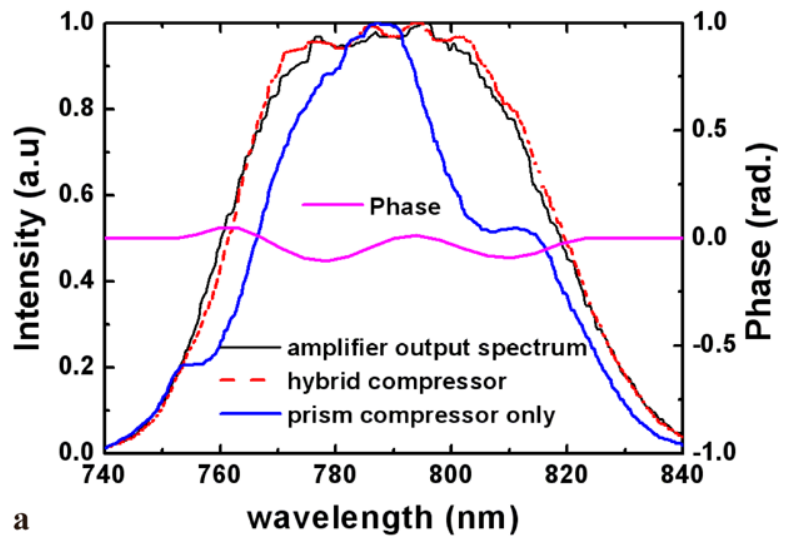

Fig. 3 a Spectrum after the amplifier (black) and HPC (red). For comparison, the spectrum of the compressed pulse (blue) is shown using the prism compressor only (previous performance). The retrieved phase (Magenta) of the compressed pulse $\left(<23 \mathrm{fs}, \mathrm{FWHM}, \mathrm{sech}^{2}\right)$

compressing them with negatively chirped HDM. We have used a total of $16 \mathrm{HDMs}$, each providing a group-delay dispersion (GDD) of $-500 \mathrm{fs}^{2}$ for a spectral range of $740-840$ $\mathrm{nm}$ with high reflectivity of more than $99.7 \%$. In order to accommodate the extra dispersion of these dispersive mirrors in our system, an additional amount of 35-mm SF57 glass is inserted in the stretcher (which is more convenient for dispersion management than reducing the prism separation). Hence the high-energy pulses still carry their positive chirp (with a pulse width $>1 \mathrm{ps}$ ) while passing through the last prism and get finally compressed by the HDMs. The higher-order dispersion of 35-mm-thick SF57 glass, i.e. third-order dispersion of $+5000 \mathrm{fs}^{3}$ and fourth-order dispersion of $+1850 \mathrm{fs}^{4}$, is compensated by the DAZZLER. The HDMs are not designed in complementary pairs, like the traditional broadband chirped mirrors, to cancel out fine phase oscillation of each other. Therefore, we observed an accumulation of $\sim 1$ rad of phase oscillations in the pulses after the compressor, using a GRENOUILLE device (Swamp Optics). These oscillations can be precompensated by loading a phase file derived from the GRENOUILLE measurement into the DAZZLER. This approach yields sub-23-fs $1.5-\mathrm{mJ}$ pulses with a spectral FWHM of $\sim 60 \mathrm{~nm}$, free from SPM effects (see Fig. 3).

In order to generate a few-cycle supercontinuum with these pulses, which is suitable for seeding the OPCPA in the NIR, we have tested the following cascaded spectral broadening schemes: (1) filamentation followed by broadening in an HCF (HCF-Filament) and (2) double-HCF approach. In the following we report on our results of these two schemes.

\subsection{Cascaded spectral broadening in HCF-filament}

The Femtopower output after the HPC with $1.5-\mathrm{mJ}$ pulse energy is focused by an $f=2 \mathrm{~m}$ lens into a $300-\mu \mathrm{m}$-inner

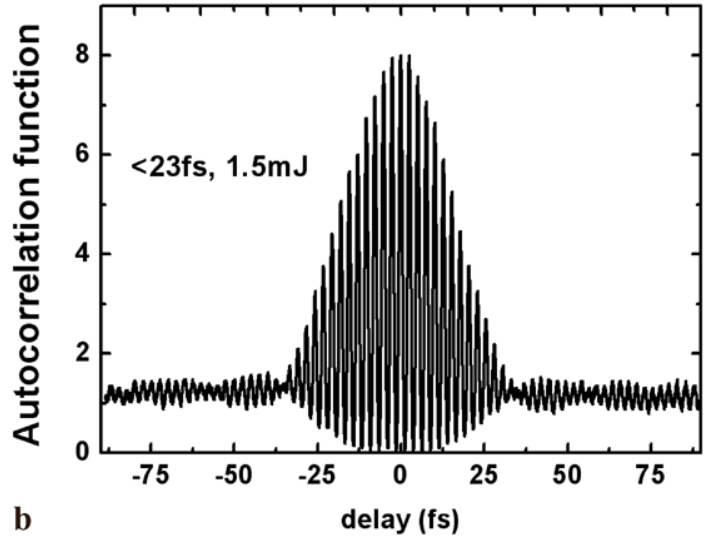

by the GRENOUILLE after the HPC is also shown. b The measured second-order interferometric autocorrelation (FWHM 15 fringes) of the compressed pulse after the HPC

diameter and 1-m-long HCF (HCF1). The pressure of Neon $(\mathrm{Ne})$ in HCF1 and the GDD of the input pulse has been adjusted to get maximum broadening. At an optimum pressure of $2.3 \mathrm{bar}$, an overall throughput of $650 \mu \mathrm{J}$ is obtained with central mode containing more than $85 \%$ energy. The pointing fluctuations at the entrance, which arise mainly from the air turbulences, mechanical instability of the mirror mounts, and the long-term thermal drift of the laser cavity, result in an instability of the HCF output and are therefore minimized by using a laser beam pointing stabilization system [BeamLock-4D,TEM-Messtechnik GmbH]. This system provides both the position and angle locking of the laser beam with four degrees of freedom in space using two position-sensitive detectors via an active feedback loop control and hence stabilizes the throughput of HCF in terms of the beam profile, output spectrum, and energy throughout each working day.

The output of HCF1 is collimated by a $0.75-\mathrm{m}$ focal length silver-coated mirror and then compressed using a negatively chirped mirror compressor. These mirrors have been designed in-house to provide a flat GDD of $-40 \mathrm{fs}^{2}$ per reflection in a broad spectral range of 550-1000 nm. After HCF1 the output spectrum is compressed down to $\sim 4.2$ fs and then focused into an Argon-cell (Ar-cell) by using a 2-m focal length silver-coated mirror at a pressure of $700 \mathrm{mbar}$ with a $1 / \mathrm{e}^{2}$ beam diameter of $350 \mu \mathrm{m}$. In order to fine control the spatial beam profile at the output of the Ar-cell, an iris was placed into the input beam of the Arcell. We observed the onset of multiple filamentation even at sub-atmospheric pressures of Ar due to the high intensity of the input pulse. Fine adjustment of the iris at 750-mbar pressure of Ar helped in obtaining a good quality spatial beam profile in a single filament. Under these conditions, we are able to couple $\sim 400 \mu \mathrm{J}$ of the input pulse energy into the Arfilament. A broadband supercontinuum containing $\sim 100-\mu \mathrm{J}$ 

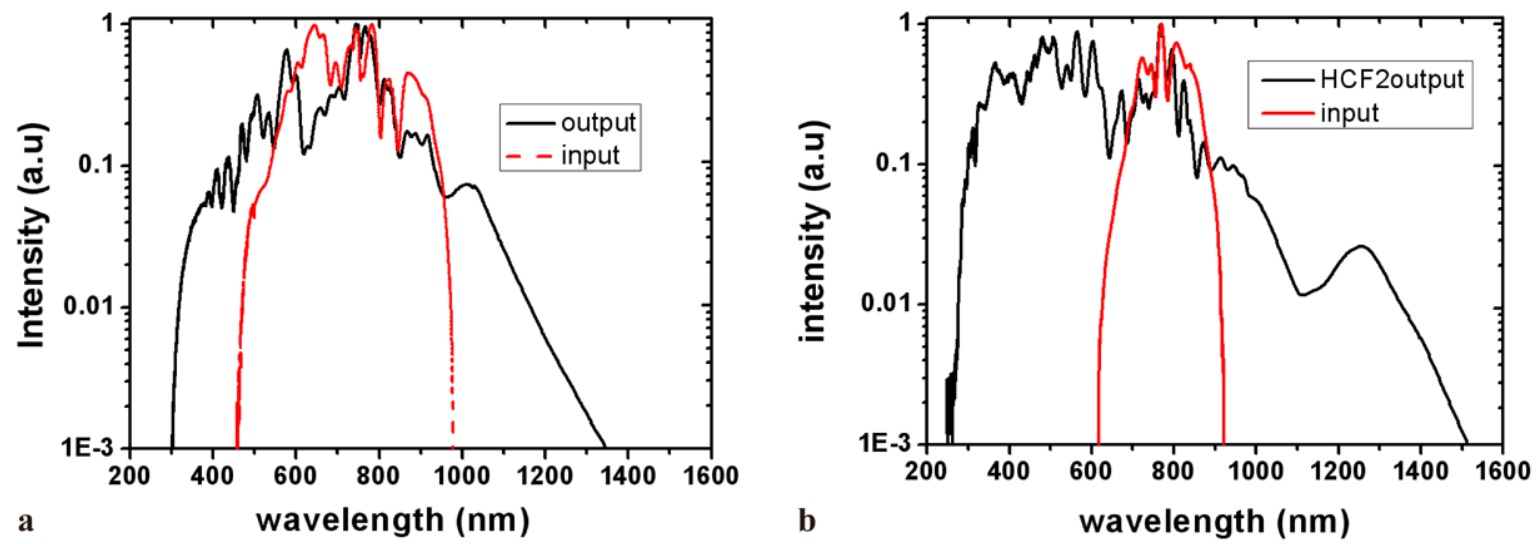

Fig. 4 a HCF-Filament: Input and output spectrum of the Argon cell at a pressure of 750 mbar. b Double-HCF: input and output spectrum of the $\mathrm{HCF} 2$ at a pressure of 3.3 bar of $\mathrm{Ne}$

energy in the central mode is generated after the cell (see Fig. 4a). The broadband supercontinuum is measured using AvaSpec-3648 and AvaSpec-NIR256-1.7 (Avantes) spectrometers. A 2.3-fs Fourier-limited pulse duration can be inferred from this spectrum, centered at $600 \mathrm{~nm}$. About 55\% of the supercontinuum energy is contained in the spectral range of 700-1400 nm.

\subsection{Cascaded spectral broadening in double-HCF}

As an alternative, we replaced the Ar-cell by another $\mathrm{HCF}$ (HCF2) as shown in Fig. 1. In this case the output spectrum of HCF1 was not set for maximum broadening but for reduced spectral modulations and hence good compression with minimal pre-pulse or post-pulse wings, while keeping a sufficient bandwidth for $\sim 5$-fs pulses. This was achieved by fine tuning of the GDD in the DAZZLER and by changing the gas pressure. The optimum pressure of $\mathrm{Ne}$ was found to be 1.5 bar. The overall throughput of HCF1 was similar to that described above. The output was compressed down to $\sim 5.0 \mathrm{fs}$ and then focused by an $f=1.0 \mathrm{~m}$ silver mirror into the 1-m long HCF2 with inner diameter $250 \mu \mathrm{m}$ at a pressure of 3.3 bar of Ne. At the output of HCF2, the highenergy supercontinuum $(200 \mu \mathrm{J})$ extends up to nearly three octaves $(\sim 260-1500 \mathrm{~nm})$ at the level of 3 orders of magnitude (as shown in Fig. 4b). Our Fourier transform calculations show that this broadband spectrum supports a pulse width of $1.3 \mathrm{fs}$ centered at $550 \mathrm{~nm}$. About $25 \%$ of the total energy is contained in the spectral range $(700-1400 \mathrm{~nm})$ required for seeding the main OPCPA chain in the PFS.

\subsection{Theoretical calculations of short-pulse pumped OPCPA}

In order to verify the suitability of the NIR tail of these supercontinua for seeding the main OPCPA chain of the PFS system, we numerically modeled the OPCPA stages using a simplified 1D-approximation [27, 28]. The experimentally measured spectra in both cases (HCF-Filament and doubleHCF scheme) were used as the input of the first stage. The pump pulse and the OPCPA seed pulse duration was taken to be $1.5 \mathrm{ps}$ (FWHM) and $1 \mathrm{ps}$ (FWHM), respectively. The anticipated total pump energy of $20 \mathrm{~J}$ at $515 \mathrm{~nm}$ was divided into seven amplification stages ranging from $2 \mathrm{~mJ}$ to $5 \mathrm{~J}$, while keeping the energy fluence well below the measured damage threshold $\left(\sim 700 \mathrm{~mJ} / \mathrm{cm}^{2}\right)$ of the available DKDP crystals. The crystal thickness of the DKDP was assumed to range from $3.5 \mathrm{~mm}$ (for the 1st stage) to $1 \mathrm{~mm}$ (for the 7th stage) and a noncollinear type-I geometry was used with a phase-matching angle of $37^{\circ}$ and a pump-seed angle of $1^{\circ}$. The calculations show that in a series of seven amplification stages, the seed pulses in the spectral range $(700-1400 \mathrm{~nm})$ can reach the 2-3 J level. The seed spectrum and the resulting amplified output spectra of the first and the last (7th) stages as obtained by the calculations are depicted in Fig. 5 . It can be seen that the modulations in the seed spectrum, for both cases described above, are smoothened until the final stage and the calculated Fourier limited pulse duration supported by the spectrum after the 7th stage is below $5 \mathrm{fs}$. We can infer from these calculations that both the schemes are suitable for seeding the OPCPA in the NIR. The HCFFilament scheme is attractive due to the ease of alignment. However, it requires more careful control on the gas pressure and input pulse energy. On the other hand, the double-HCF scheme seems more attractive due to the higher energy in the NIR, which is better suited for OPCPA amplification. The stringent requirement of alignment of the HCF2 is relaxed by installing a $4 \mathrm{D}$ laser beam pointing stabilization system at HCF1 input (as discussed earlier in Sect. 4.2). Therefore we adopt the double-HCF scheme for seeding the OPCPA chain in PFS.

In order to obtain efficient amplification the seed pulses need to be stretched so that they match the pump duration. In the following we present the details about the stretching scheme. 


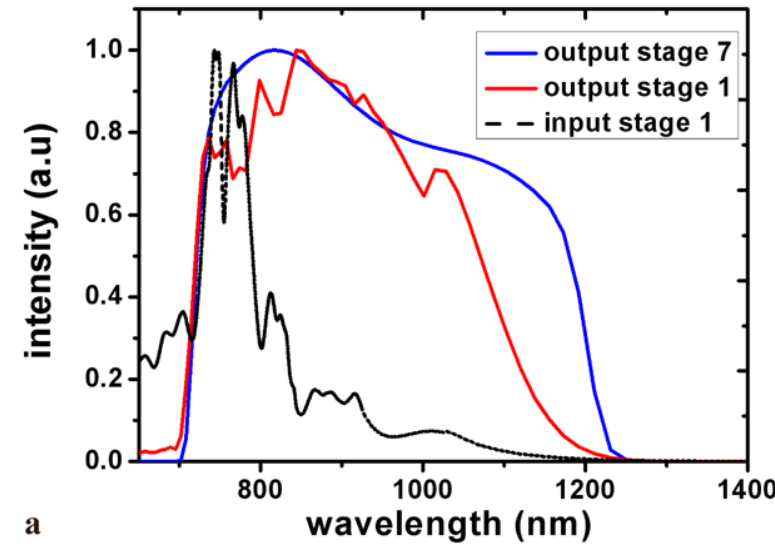

Fig. 5 Numerically calculated OPCPA amplified spectrum of the first and the last (7th) stage, using: a HCF-Filament output spectrum. b Double-HCF output spectrum as input of the first stage. All spectra

\subsection{OPCPA seed stretcher}

We use a negative dispersion paired double-prism stretcher to stretch the OPCPA seed pulses. The pulses will therefore be amplified with negative chirp and compressed in bulk material alone or in combination with chirped mirrors. The design of the stretcher follows the equations given in Ref. [29], in order to select the prism material and the required separation between the prism pairs. Here we assume that the duration of the supercontinuum pulses after HCF2 closely matches to that of the input pulses [20, 22]. Fused silica prisms have been selected because of their small highorder group-velocity dispersion in the spectral range of 700$1400 \mathrm{~nm}$ as compared to other common prism materials (like SF10, SF14, LaK21) and the relaxed requirements for total second-order dispersion. A prism pair separation of $\sim 40 \mathrm{~cm}$ (apex to apex) yields a stretched duration of 2 ps over the whole spectral range. The experimental setup is shown in Fig. 1. The desired part of the spectrum $(700-1400 \mathrm{~nm})$ from the supercontinuum was selected by clipping the dispersed spectrum, after the first prism, on the apex of the second prism pair at $680 \mathrm{~nm}$. This technique seems to be a convenient way of precisely controlling the amount of dispersion for our broad spectral range on a day-to-day basis. The position of the resulting spectral clip is easily monitored and is a good indication for the amount of inserted prism material. The stretched pulses are characterized by a modified frequency-domain phase measurement (FDPM) scheme [30] by measuring their cross-correlation with a fraction of the Femtopower output $(60 \mu \mathrm{J})$ as the reference, which is also schematically shown in Fig. 1. The measured groupdelay (GD) of the 2-ps pulse after the prism stretcher is depicted in Fig. 6, for an apex-apex separation of $40 \mathrm{~cm}$ between the prism pairs. In order to finally control the pulse duration and phase of the seed pulses, we plan to use ultra

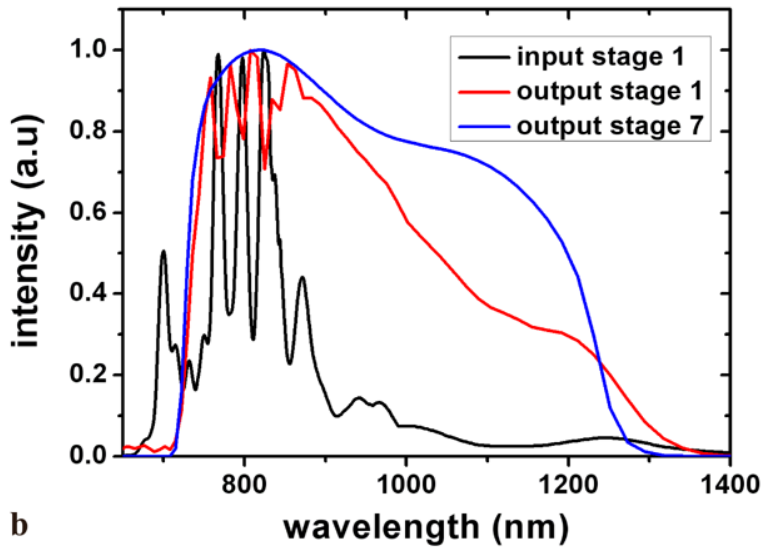

have been normalized to their respective maxima in order to show the amplification bandwidth and the amplitude of the modulations more clearly

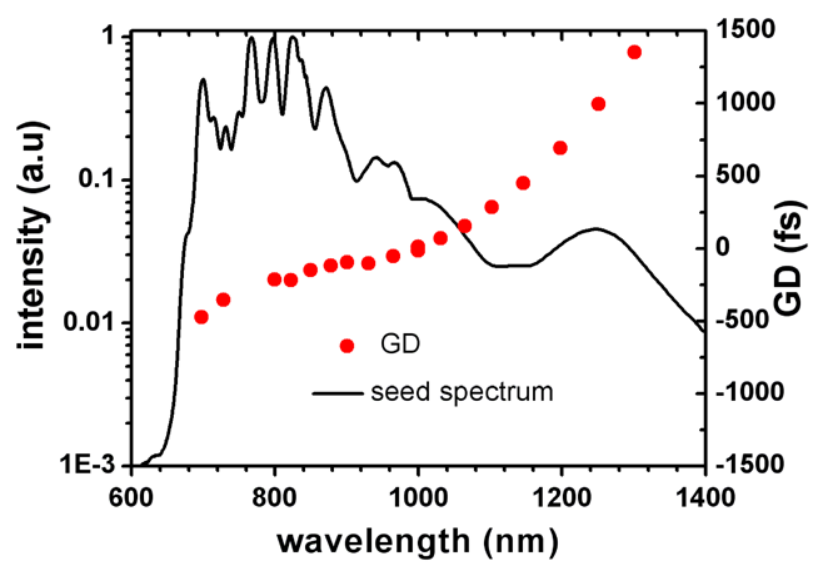

Fig. 6 FDPM measurement of the OPCPA seed

broadband chirped mirrors, which are currently being designed. Our aim is to adjust the phase of the seed pulses in a way that allows for compression in bulk material at the end of the amplification chain.

\section{Temporal synchronization between the pump laser and OPCPA seed}

In order to quantify the level of synchronization between the seed and pump pulses in a real OPCPA experiment, we measured the scanning cross correlation between the Femtopower $(<30 \mathrm{fs}, 40 \mu \mathrm{J})$ and the RA output pulses which simulate the time position of the OPCPA seed and pump laser pulses, respectively. The scheme of synchronization is shown in Fig. 7a. Different optical pulses from the MO have been selected for the OPCPA-seed and pump laser seed in order to compensate the long delay of about $300 \mathrm{~ns}$ due to the 40 round-trips in the RA. For this measurement, the 


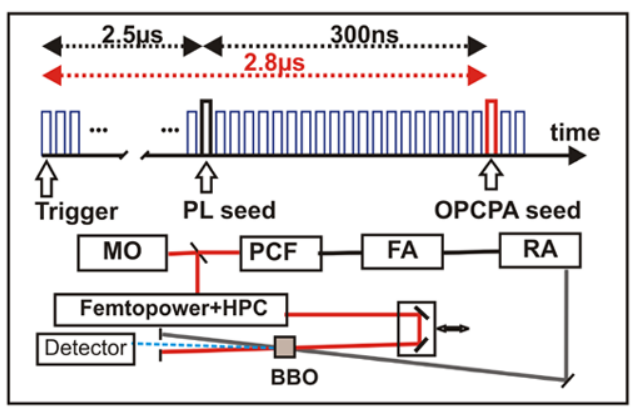

a

Fig. 7 a The scheme of measuring the cross correlation in order to quantify the temporal synchronization between the pump laser and OPCPA seed. RA: regenerative amplifier; FA: fiber amplifier; MO: master oscillator; PCF: photonic crystal fiber; PL seed: (CPA) pump-

unstretched pump laser seed pulses from the fiber amplifier were directly amplified in the Yb:glass RA to about $10 \mu \mathrm{J}$ $(10 \mathrm{~Hz}, 1030 \mathrm{~nm}, 4.4 \mathrm{ps})$. This technique of by-passing the stretcher is necessary to achieve sub-ps resolution. The cross correlation between the two pulses was recorded by observing the SFG signal in a $100-\mu$ m-thick BBO crystal, and the measurements were repeated at different times over a full duration of about four hours. The position of the peaks of the SFG signal provides a direct measurement of the delay of one pulse with respect to the other. We fitted a Gaussian to each of the cross correlation curves in order to determine the relative position of the pump and seed pulses. The peaks of these Gaussian fits are shown as functions of the time of measurement in Fig. 7b. For about 150 minutes from the start of the experiment, we observed a drift of about 3 ps between the two pulses, which later reached a steady state with a short-term drift of less than $100 \mathrm{fs}$ (inset of Fig. 7b). From the stability of the peak position we estimate the resolution of our measurement to be on the order of $100 \mathrm{fs}$; therefore this result represents an upper limit of the actual temporal drift. During the measurement, we also recorded the temperature at the surface and underneath the optical tables. We observed a temperature change of about $0.9 \mathrm{~K}$ at the surface of the optical table during early 2-3 hours of laser operation. Later, the table surface temperature was quite stable within $\pm 0.1 \mathrm{~K}$. The temperature change during these early (2-3) hours of laser operation was associated with the temperature gradient under the tables due to the warm-up of the pump laser power supplies. The slow drift during the first three hours can be explained by this temperature change. In our setup the estimated difference in the optical paths of the two pulses was about $75-80 \mathrm{~m}$. By considering the linear thermal expansion coefficient of stainless steel of $13 \times 10^{-6} \mathrm{~K}^{-1}$, a temperature change of $1 \mathrm{~K}$ for a path difference of $70 \mathrm{~m}$ between the two pulses causes a drift of $\sim 3 \mathrm{ps}$. Therefore, under current operating conditions, a simple solution of this

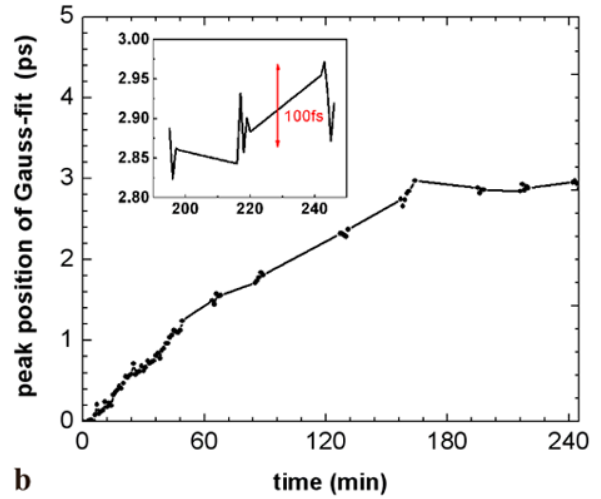

laser seed; HPC: hybrid pulse compressor. b Measured time position of maxima with respect to the time of measurement. In the inset is shown the short-term temporal drift ( $<100 \mathrm{fs}$ ) at steady state temperature conditions

thermal drift is to leave the setup running for few hours before the start of experiment to reach stable temperature conditions. The other way is active stabilization using a balanced cross correlation technique [31].

It is necessary to mention that these measurements do not provide direct information about pulse-to-pulse time jitter, since the scans for each peak position took several minutes. The value of jitter $\tau_{\mathrm{j}}$ can in principle be estimated, to a first approximation, from the measured cross correlation width $\tau_{X}$ by the formula $\left(\tau_{\mathrm{j}}=\sqrt{\tau_{X}^{2}-\tau_{\mathrm{p}}^{2}-\tau_{\mathrm{s}}^{2}}\right)$, where $\tau_{\mathrm{p}}$ and $\tau_{\mathrm{s}}$ are the FWHM pulse durations of the pump and seed signals, respectively. As the measured $\tau_{X} \approx \tau_{\mathrm{p}}=4.4 \pm 0.1 \mathrm{ps}$, from the formula we can derive only the upper limit of $\tau_{\mathrm{j}} \leq 1 \mathrm{ps}$. More precise estimation would require this experiment to be performed with the compressed pump pulses on $\sim 1$-psscale, which is beyond the scope this paper.

\section{Conclusion}

We have presented the development of a light source which serves as a frontend of the PFS system based on ps-scale OPCPA. The stringent requirement for synchronization between the seed pulses of the CPA pump laser chain and the main OPCPA chain has been fulfilled by deriving both of these pulses from a common "master oscillator" source, thereby providing optical synchronization. A short-term temporal drift on a time scale of less than $100 \mathrm{fs}$ has been observed, which will allow for sufficient synchronization in the short-pulse pumped OPCPA scheme, keeping the amplification conditions constant over extended periods of time, as was already reported in our proof of principle experiment [28]. Starting from the MO pulses, the seed for the pump laser chain at $1030 \mathrm{~nm}$ is generated by soliton 
based spectral shifting. The broadband OPCPA seed spectrum $(700-1400 \mathrm{~nm})$ is obtained using the nonlinear interaction of the few-cycle pulses with gaseous media, where we have presented the HCF-filament and double-HCF schemes for supercontinuum generation. Our simplified 1D-model simulation shows that the NIR tail of the spectra from both of these schemes can be amplified to PW-scale in DKDP using ps-timescale amplification. The choice of double-HCF is taken due to the better shape of the spectrum in the NIR. Using this frontend system in combination with the recent ongoing progress in developing the few-ps pump source $[14,19]$ will pave the way towards the ambitious goals of the PFS system.

Acknowledgements This work is funded through the PFS grant of the Max-Planck Society. I. Ahmad thanks for the HEC-DAAD 2006 fellowship. Partial financial support from the Extreme Light Infrastructure (ELI) and the DFG Cluster of Excellence (Munich Centre for Advanced Photonics) is also acknowledged.

Open Access This article is distributed under the terms of the Creative Commons Attribution Noncommercial License which permits any noncommercial use, distribution, and reproduction in any medium, provided the original author(s) and source are credited.

\section{References}

1. J. Faure, Y. Glinec, A. Pukhov, S. Kiselev, S. Gordienko, E. Lefebvre, J.P. Rousseau, F. Burgy, V. Malka, Nature 431, 541 (2004)

2. S.P.D. Mangles, C.D. Murphy, Z. Najmudin, A.G.R. Thomas, J.L. Collier, A.E. Dangor, E.J. Divall, P.S. Foster, J.G. Gallager, C.J. Hooker, D.A. Jaroszinsky, A.J. Lagley, W.B. Mori, P.A. Norreys, F.S. Tsung, R. Viskup, B.R. Walton, K. Krushelnick, Nature 431, 535 (2004)

3. C.G.R. Geddes, C. Toth, J. van Tilborg, E. Esarey, C.B. Schroeder, D. Bruhwiler, C. Nieter, J. Cary, W.P. Leemans, Nature 431, 538 (2004)

4. M. Geissler, J. Schreiber, J. Meyer-ter-Vehn, New J. Phys. 8, 186 (2006)

5. Y. Nomura, R. Horlein, P. Tzallas, B. Dromey, S. Rykovanov, Zs. Major, J. Osterhoff, S. Karsch, L. Veisz, M. Zepf, D. Charalambidis, F. Krausz, G.D. Tsakiris, Nat. Phys. 5, 124 (2009)

6. A. Dubietis, G. Jonušauskas, A. Piskarskas, Opt. Commun. 88, 437 (1992)

7. N. Ishii, L. Turi, V.S. Yakovlev, T. Fuji, F. Krausz, A. Baltuška, R. Butkus, G. Veitas, V. Smilgevičius, R. Danielius, A. Piskarskas, Opt. Lett. 30, 567 (2005)

8. O.V. Chekhlov, J.L. Collier, I.N. Ross, P.K. Bates, M. Notley, C. Hernandez-Gomez, W. Shaikh, C.N. Danson, D. Neely, P. Matousek, S. Hancock, L. Cardoso, Opt. Lett. 31, 3665 (2006)

9. V.V. Lozhkarev, G.I. Freidman, V.N. Ginzburg, E.V. Katin, E.A. Khazanov, A.V. Kirsanov, G.A. Luchinin, A.N. Mal'shakov, M.A.
Martyanov, O.V. Palashov, A.K. Poteomkin, A.M. Sergeev, A.A. Shaykin, I.V. Yakovlev, Laser Phys. Lett. 4, 421 (2007)

10. F. Tavella, A. Marcinkevicius, F. Krausz, Opt. Express 14, 12822 (2006)

11. S. Karsch, Zs. Major, J. Fülöp, I. Ahmad, T.-J. Wang, A. Henig, S. Kruber, R. Weingartner, M. Siebold, J. Hein, C. Wandt, S. Klingebiel, J. Osterhoff, R. Hörlein, F. Krausz, in Advanced Solid-State Photonics, OSA Technical Digest Series (CD) (Optical Society of America, Washington, 2008), paper WF1

12. Zs. Major, S.A. Trushin, I. Ahmad, M. Siebold, C. Wandt, S. Klingebiel, T.-J. Wang, J.A. Fülöp, A. Henig, S. Kruber, R. Weingartner, A. Popp, J. Osterhoff, R. Hörlein, J. Hein, V. Pervak, A. Apolonski, F. Krausz, S. Karsch, Rev. Laser Eng. 37 (2009, in press)

13. D. Strickland, G. Mourou, Opt. Commun. 56, 219 (1985)

14. M. Siebold, Ch. Wandt, S. Klingebiel, Zs. Major, S.A. Trushin, I. Ahmad, T.-J. Wang, J. Hein, F. Krausz, S. Karsch, Proc. SPIE 6998, 69980E (2008)

15. F. Tavella, A. Marcinkevičius, F. Krausz, New J. Phys. 8, 219 (2006)

16. N. Ishii, C.Y. Teisset, T. Fuji, S. Köhler, K. Schmid, L. Veisz, A. Baltuska, F. Krausz, IEEE J. QE. 12, 173 (2006)

17. C.Y. Teisset, N. Ishii, T. Fuji, T. Metzger, S. Köhler, R. Holzwarth, A. Baltuška, A.M. Zheltikov, F. Krausz, Opt. Express 13, 6550 (2005)

18. P.S. Banks, M.D. Perry, V. Yanovsky, S.N. Fochs, B.C. Stuart, J. Zweiback, IEEE J. QE. 36, 268 (2000)

19. C. Wandt, S. Klingebiel, M. Siebold, Zs. Major, J. Hein, F. Krausz, S. Karsch, Opt. Lett. 33, 1111 (2008)

20. S.A. Trushin, K. Kosma, W. Fuß, W.E. Schmid, Opt. Lett. 32, 2432 (2007)

21. N. Aközbek, S.A. Trushin, A. Baltuška, W. Fuß, E. Goulielmakis, K. Kosma, F. Krausz, S. Panja, M. Uiberacker, W.E. Schmid, A. Becker, M. Scalora, M. Bloemer, New J. Phys. 8, 177 (2006)

22. E. Goulielmakis, S. Koehler, B. Reiter, M. Schultze, A.J. Verhoef, E.E. Serebryannikov, A.M. Zheltikov, F. Krausz, Opt. Lett. 33, 1407 (2008)

23. B. Schenkel, J. Biegert, U. Keller, C. Vozzi, M. Nisoli, G. Sansone, S. Stagira, S. De Silvestri, O. Svelto, Opt. Lett. 28, 1987 (2003)

24. S.A. Planas, N.L.P. Mansur, C.H.B. Cruz, H.L. Fragnito, Opt. Lett. 18, 699 (1993)

25. A.L. Cavalieri, E. Goulielmakis, B. Horvath, W. Helml, M. Schultze, M. Fiess, V. Pervak, L. Veisz, V.S. Yakovlev, M. Uiberacker, A. Apolonski, F. Krausz, R. Kienberger, New J. Phys. 9, 242 (2007)

26. V. Pervak, C. Teisset, A. Sugita, S. Naumov, F. Krausz, A. Apolonski, Opt. Express 16, 10220 (2008)

27. G. Cerullo, S. De Silvestri, Rev. Sci. Instrum. 74, 1 (2003)

28. J.A. Fülöp, Zs. Major, A. Henig, S. Kruber, R. Weingartner, T. Clausnitzer, E.-B. Kley, A. Tünnermann, J. Osterhoff, R. Hörlein, F. Krausz, S. Karsch, New J. Phys. 9, 438 (2007)

29. Z. Cheng, F. Krausz, Ch. Spielmann, Opt. Commun. 201, 145 (2002)

30. J.L.A. Chilla, O.E. Martinez, IEEE J. QE. 27, 1228 (1991)

31. T.R. Schibli, J. Kim, O. Kuzucu, J.T. Gopinath, S.N. Tandon, G.S. Petrich, L.A. Kolodziejski, J.G. Fujimoto, E.P. Ippen, F.X. Kaertner, Opt. Lett. 28, 947 (2003) 